\title{
Adaptive Fair Resource Allocation and Packet Dependent Scheduling Using Cross-Layer Design in Multiuser OFDM Systems
}

\author{
Kalpana Goyal, Member, IACSIT, Aditya Trivedi, and Karan Nathwani, Member, IACSIT
}

\begin{abstract}
In this paper, we consider a multiuser orthogonal frequency division multiplexing (OFDM) system and propose a fair resource allocation algorithm using cross-layer design with packet dependent scheduling. Our proposed algorithm achieves fairness among users (in proportion of weights of users) while maximizing the weighted sum capacity of users. Since the optimal solution to the problem is computationally complex to obtain, a low-complexity suboptimal algorithm is proposed. It is assumed that each user is having multiple heterogeneous traffic queues. It is a user based resource allocation algorithm where weight of user can be calculated by summing up the weight of all packets in all the queues of that user. A packet dependent scheduling scheme is also used at medium access control (MAC) layer to determine the packet transmission order according to their weights. The weight of a packet is decided by its size, delay tolerance, and quality of service $(\mathrm{QoS})$ priority level.
\end{abstract}

Index Terms-Cross-layer design, dynamic resource allocation, fairness, OFDM, packet scheduling, quality of service $(\mathrm{QoS})$.

\section{INTRODUCTION}

To fulfill the increasing requirements for high speed data transmission and efficient resource management, orthogonal frequeny division multiplexing (OFDM) is a promising technique for the next generation of wireless communication systems. OFDM divides a broadband channel into a set of orthogonal narrowband subcarriers. It is used to combat frequency selective fading and to avoid intersymbol interference. OFDM transforms frequency selective fading to flat fading [1][2][3].

To support multiuser multi-tasking services, efficient use of radio resource is needed. Hence adaptive resource management scheme should be employed to dynamically allocate bandwidth to the users [4]. The resource management scheme should be efficient and fair in utilizing the radio resources. Conventional communication networks (where each layer operates independently) do not utilize the spectrum and energy efficiently. Hence a cross-layer design of the wireless networks is desirable. So a packet dependent

Manuscript received January 30, 2012; revised March 4, 2012. This work was supported by ABV-IIITM Gwalior.

Kalpana Goyal is with the Department of Electronics, Maulana Azad National Institue of Technology, Bhopal, India (e-mail: kalpana.goyal19@gmail.com).

Aditya Trivedi is with the Department of ICT, ABV-Indian Institute of Information Technology and Management, Gwalior, India (e-mail: atrivedi@iiitm.ac.in).

Karan Nathwani is with the Indian Institue of Technology, Kanpur(e-mail: karan046@gmail.com). scheduling is also required at medium access control (MAC) layer which calculates the weights of the packets. The packet scheduler collects the cross-layer information (channel state information and queue state information) and select different users' packets for transmission according to the information $[5]$.

Much research work has been carried out on utilizing the cross-layer information for resource allocation and/or packet scheduling. In [2], resource allocation is performed based on a utility function which reflects the quality of service and it also includes multichannel scheduling that exploits wireless channel and queue information. However, queue based scheduling is considered in this paper, which leads to inefficiency. In [3], a resource allocation scheme is developed by integrating power distribution, subcarrier allocation, and generalized processor sharing (GPS) scheduling to maximize system throughput and provides fairness to all traffic. In [5], a quality of service (QoS) aware proportional fair packet scheduling algorithm is proposed to allocate radio resources among users, based on cross-layer design in that scheduler is aware of both channel and queue state information. However in [3] and [5], it is assumed that each user obtains a single traffic queue and each queue is having homogeneous traffic. In [6], a resource allocation framework is proposed which maximizes the sum capacity while maintaining proportional fairness among users. In [7], a multiuser subcarrier, bit, and power allocation scheme is proposed where all users transmit in all the time slots and objective of this algorithm is to minimize the overall transmit power of an OFDM system with fixed data rate. But, the work in [6] and [7] did not consider cross-layer design. In [8], advantages of a cross-layer approach to the resource allocation algorithm are discussed. A maximum delay utility (MDU) based cross-layer design is proposed in [9] which performs resource allocation and scheduling by maximizing a utility function of the delay. A modified largest weighted delay first (M-LWDF) scheduling scheme was proposed for the MAC layer of a single carrier system in [10], which assigns a higher priority to the queues that have a larger head-of-line (HOL) packet delay relative to the delay bound, a higher instantaneous data rate relative to the average data rate, and a higher requirement for the outage probability. But the M-LWDF and MDU scheduling schemes are queue based scheduling that is not efficient as packet dependent scheduling.

In [4], an adaptive cross-layer design for the downlink multiuser OFDM systems is proposed, having multiple heterogeneous traffic queues for all users. This maximizes 
the weighted sum capacity of all users and also employed packet dependent scheduling scheme at MAC layer but in this paper fairness is not considered.

In this paper, an adaptive cross-layer design is proposed which maximizes the weighted sum capacity of OFDM systems and also includes fairness criteria. Here fairness implies that users will get the data rate in proportion of their weights. Fairness is considered due to the reason that users with high channel gains will be allocated most of the resources and users with low channel gains will not be able to receive any data [6]. In [4], fairness criteria are not considered. We modified the algorithm of [4] that balances trade-off between maximum weighted sum capacity (MWSC) and fairness. Here, we considered multiple heterogeneous traffic queues for all users. A packet dependent scheduling scheme is also employed at MAC layer which calculates the weight of packets and transmits the packets in the order of their weights. The weight of a packet is calculated by the delay, size, and QoS priority level of packets. We have proposed a user based resource allocation algorithm because it has lower complexity than queue based resource allocation. Simulation results show that MWSC resource allocation algorithm with fairness has better performance in terms of throughput and delay as compared to MWSC resource allocation algorithm without fairness but the complexity (for comparisons) of the modified algorithm is $O\left(N^{2}\right)$ comparisons while the complexity of algorithm in [4] is $O(K N)$, where $N$ denotes the number of subcarriers and $\mathrm{K}$ denotes the number of users. Since $N>K$, hence complexity increases.

This paper is organised as follows. Section II introduces the Multiuser OFDM system model and problem formulation. Section III shows the suboptimal MWSC based fair resource allocation algorithm. Section IV presents the packet dependent data scheduling. Section V shows simulation results and section VI draws the conclusion.

\section{SYSTEM MODEL AND PROBLEM FORMULATION}

\section{A. System Model}

We consider a downlink multiuser OFDM system where each subcarrier has a bandwidth smaller than the coherence bandwidth of the channel and instantaneous channel gains on all the subcarriers of all users are known to the transmitter. The channel considered is quasi-static fading channel, where the channel gain is constant during each slot.

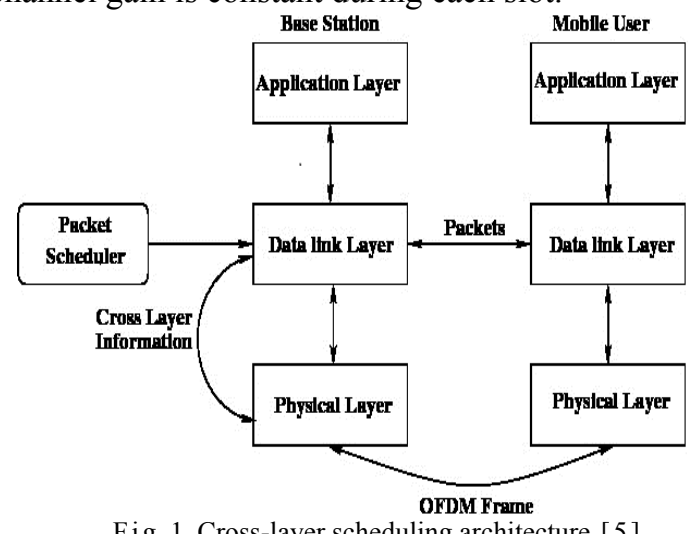

Fig.1.Cross-layer scheduling architecture [5]
The cross-layer scheduling architecture [5] is shown in Fig. 1. The OFDM frames are transmitted through physical layers between the base station and mobile user. The subcarrier and power controller at the physical (PHY) layer performs subcarrier and power allocation, and the traffic controller at the MAC layer performs data scheduling. With a cross-layer design, the resource allocation results are transferred to MAC layer for scheduling of data and QoS information is transferred to physical layer.

Assuming a total of $K$ users in the system having $N$ independent subcarriers and total bandwidth of $B$. The total transmit power is $P_{\text {Total }}$. The OFDM signaling is time slotted and length of a time slot is $T_{\text {slot }}$ [2]. Assume that each user has $I_{k}$ traffic queues with heterogeneous delay constraints. Let $S_{k}$ denotes the subcarrier index set allocated to user $k \quad(k \in\{1, \ldots \ldots \ldots . . . K\})$. Assume that $P_{k, n}$ denotes the power allocated to user $k$ on subcarrier $n, h_{k, n}$ is the corresponding channel gain and $N_{o}$ is the power spectral density of additive white gaussian noise (AWGN). Assuming perfect channel estimation, the achievable instantaneous data rate of user $k$ on subcarrier $n$ is given by:

$$
R_{k, n}=\frac{B}{N} \log _{2}\left(1+P_{k, n} \gamma_{k, n}\right)
$$

where

$$
\gamma_{k, n}=\frac{h_{k, n}^{2}}{N_{0} B / N}
$$

is the channel-to-noise power ratio for user $k$ on subcarrier $n$. The total achievable instantaneous data rate of user $k$ is given by:

$$
R_{k}=\sum_{n \in S_{k}} R_{k, n}
$$

\section{B. Problem Formulation}

We employ a MWSC based cross-layer design which maximizes the weighted sum capacity and also achieves fairness among users. Let $W_{k}$ denoted the weight for user $k$, which is determined by packet scheduling algorithm at MAC layer. The cost function to be maximized is given by:

$$
J=\sum_{k=1}^{K} W_{k} R_{k}
$$

Subject to the constraints:

$$
\begin{aligned}
& \text { (C1) } R_{1}: R_{2}: \ldots \ldots: \not R_{k}=W_{1}: W_{2}: \ldots \ldots: W_{k} \\
& \text { (C2) } P_{k, n} \geq 0 \\
& \text { (C3) } \sum_{k=1}^{K} \sum_{n \in S_{k}} P_{k, n} \leq P_{\text {Total }} \\
& \text { (C4) } R_{k} T_{\text {slot }} \leq Q_{k} \\
& \text { (C5) } S_{1} \cup \ldots \ldots \cup S_{K} \subseteq\{1,2, \ldots \ldots ., N\} \\
& \text { (C6) } S_{k} \cap S_{j}=\varnothing(k \neq j)
\end{aligned}
$$

Constraint (C1) guarantees the fairness of algorithm it implies that all users get the data rate in the proportion of their weights. Algorithm in [4] is not subjected to constraint (C1). Constraint (C4) implies that if any user obtains sufficient resources to send all data in a particular 
time slot then after that resources will not be allocated to that user to avoid wastage of bandwidth and constraint (C6) guarantees that each subcarrier is allocated to only one user [2][8].

\section{Suboptimal Fair Maximum Weighted Sum CAPACITY BASED RESOURCE AlLOCATION AlgORITHM}

To achieve the optimal solution [11], joint subcarrier and power allocation should be carried out but this increases the complexity. So to make a trade-off between performance and complexity, we propose a modified suboptimal algorithm which performs subcarrier and power allocation separately which reduces the complexity. Low complexity algorithms are preferred for cost-effective and delay-sensitive implementations.

First we perform subcarrier allocation assuming equal power distribution across all subcarriers, and then perform power al- location. In the proposed algorithm, at each iteration subcarrier allocation algorithm picks out the user having highest $W_{k} / R_{k}$ ratio and assign subcarrier $n$ to user $k$ satisfying $R_{k, n}>R_{k, j}$ where $j \in\{1,2, \ldots . N\}$. Let $U$ denotes the user index set and $S C$ denotes the subcarrier index set. The dynamic algorithm to implement subcarrier and power allocation is described as follows:

1) Initialization: Set $U=\{1,2, \ldots ., K\}, S C=\{1,2, \ldots \ldots, N\}, R_{k}$ $=0, S_{k}=\varnothing, \forall k \in U$ and $P_{k, n}=P_{\text {Total }} / N(\forall n \in S C, \forall k$ $\in U)$.

2) For $k=1$ to $K$, find $\mathrm{n}$ satisfying $R k, n>R k_{k, j}$ for all $j \in S C$. Assign subcarrier $n$ to user $k$ then update $S_{k}=$ $S_{k} \cup\{\mathbf{n}\}, S C=S C-\{n\}$ and $R_{k}$ according to equation (1).

3) While $S C=\varnothing$

$$
\text { if } U=\varnothing \text {, }
$$

a) find $k$ satisfying $W_{k} / R_{k} \geq W_{i} / R_{i}$ for all $i \in U$;

b) for the found $k$, find $n$ satisfying $R k, n>R k, j$ for all $j \in S C$;

c) assign subcarrier $n$ to user $k$ and update $S_{k}=S_{k} \cup$ $\{\mathrm{n}\}$, $S C=S C-\{n\}$ and $R k$ according to equation (1)

d) if $R k T_{\text {slot }} \geq Q k$ i.e., user $k$ has obtained sufficient from resources for sending out all data, remove user $k$ set $U$.

4) Allocate power to subcarrier $n(\forall n \in\{1,2, \ldots ., N\})$ by

$$
P_{k, n}=\frac{W_{k}\left(P_{\text {Total }}+\sum_{m=1}^{K} \sum_{q \in S_{m}} \frac{1}{\gamma_{m, q}}\right)}{\sum_{m=1}^{K} W_{m} S_{m}}-\frac{1}{\gamma_{k, n}}
$$

5) For $\mathrm{n}=1$ to $\mathrm{N}$, if $\boldsymbol{P} \boldsymbol{k}, n \leq 0$ i.e., there is no enough power for subcarrier $n$, subcarrier $n$ will be removed from user set $S_{k}$.

6) Repeat steps (4) and (5) until $P k, n>0$ ( $\forall n \in$ $\{1,2, \ldots ., N\}, \forall k \in\{1,2, \ldots ., K\})$.

The proposed algorithm performs subcarrier and power allocation separately. Subcarrier allocation firstly allocates a subcarrier to every user. After that a user is picked out at each iteration having highest $W_{k} / R_{k}$ ratio and a subcarrier is allocated to this user satisfying $R_{k, n}>R_{k, j}$ for all $j \in S C$. The proposed algorithm performs better in terms of throughput, voice packet delay, and video packet delay while the overall complexity of comparisons for this algorithm is increased to $\mathrm{O}\left(N^{2}\right)$ from $\mathrm{O}(K N)$. This increase is noticed in subcarrier allocation while for power allocation, complexity is same as algorithm in [4].

\section{PACKet DePENDENT DATA SCHEDUling}

Packet dependent scheduling scheme [4] is more efficient and flexible than conventional queue based scheduling schemes. Conventional queue based scheduling such as MDU [9] and M- LWDF [10] assigns the same weight to all packets in a queue, and serve the selected queues until either the data or PHY layer resources are exhausted during each slot. Hence these algorithms are inefficient if some packets in the unselected queues are more urgent than some packets in the currently served queues. Therefore, a packet dependent scheduling scheme is proposed to calculate the weight of packets used in the fair MWSC based resource allocation algorithm. The packet weights are decided using the delay, packet size, and QoS priority level of the packets.

It is assumed that $Y_{k, i}$ is the delay tolerance for queue $i$ ( $i$ $\left.\in\left\{1, \ldots, I_{k}\right\}\right)$ of user $k(k \in\{1,2, \ldots \ldots, K \mathbf{\}})$. The delay tolerance of the voice, variable bit rate (VBR) video, and best effort (BE) traffic queues is set to be low, medium and high, respectively. If the waiting time of any packet is greater than its delay tolerance, then the packet will be dropped. A guard interval $G_{k, i}$ is introduced to reduce the packet drop rate. If the packet can wait only for the time equal to guard interval then it is a urgent packet and it should be given high priority. Letting $T_{c} \in[0, \infty)$ denote the current time, for the $l_{t h}$ packet that belongs to queue $i$ of user $k$ and arrives at time $T_{l} \in\left[0, T_{c}\right]$, the delay is given by $X_{k, i, l}=T_{c}-T_{l}$. Thus, the time left, after that the packet becomes urgent, is denoted by $R_{k, i, l}$ (in msec), which is expressed as:

$$
R_{k, i, l}=Y_{k, i}-X_{k, i, l}-G_{k, i}
$$

Let $\theta_{k, i, l}$ denote the size of the packet that belongs to queue $i$ of user $k$ and arrives at time $T_{l}$ and $\beta_{k, i} \in[1, \infty)$ is the QoS priority level for queue $i$ of user $k$. The weight of the to-be-served packets $W_{k, i, l}$ that belongs to queue $i$ of user $k$ and arrives at time $T_{l}$ is given by:

$$
W_{k, i, l}= \begin{cases}\frac{\beta_{k, i} \theta_{k, i, l}}{R_{k, i, l}+1} & \text { if } R_{k, i, l} \geq 0 \\ \beta_{k, i} \theta_{k, i, l} & \text { if } R_{k, i, l}<0\end{cases}
$$

Hence the packet dependent scheduling does not consider that the packets are in the same queue or in different queue, it will firstly transmit the packets having higher weights. To reassemble the packets, a unique sequence number is assigned to every packet.

The first $\Lambda_{k, i}$ to-be-served packets (most urgent packets) in queue $i$ of user $k$ are selected for weight calculation of the MWSC based cross-layer design. The $\Lambda_{k, i}$ selected packets are divided into two sets: 1) the packets falling in the guard interval, denoted by $L_{k, i}^{R} ; 2$ )the rest packets, denoted by $L_{k, i}^{R}$ Hence weight $W_{k}$ of a user for the current slot is given by: 


$$
W_{k}=\sum_{i=1}^{I_{k}} \sum_{l \in L_{k, i}^{U} \cup L_{k, i}^{R}} W_{k, i, l} .
$$

In equation (8), there should be a reasonable value of $\Lambda_{k, i}$ because if the value of $\Lambda_{k, i}$ is small then the complexity decreases but the performance of the cross-layer design is also degraded while for large valued $\Lambda_{k, i}$, the complexity increases but the performance of the cross-layer design is improved. A reasonable value of $\Lambda_{k, i}$ is below 100 .

\section{Simulation Results}

In this section, we evaluate the performance of proposed fair MWSC based resource allocation algorithm and packet dependent scheduling using cross-layer design for an OFDM system. It is assumed that OFDM system has a total transmit power of $P_{\text {Total }}=1 \mathrm{~W}$, a slot duration of $T_{\text {slot }}=2 \mathrm{msec}$, a total bandwidth of $B=5 \mathrm{MHz}$ which is divided into $N=512$ subcarriers, and power spectral density of AWGN $N_{o}=0.2 *$ $10^{-8}$.

Assume that each user has $I_{k}=3$ queues: voice, VBR video, and BE traffic queues. The delay tolerance for voice, video, and BE traffic is set to be $Y_{k, i}^{\text {voice }}=100 \mathrm{msec}, Y_{k, i}^{\text {video }}=$ $400 \mathrm{msec}$ and $Y_{k, i}^{B E}=1000 \mathrm{msec}$ respectively and the guard interval $G_{k, i}$ is $10 \mathrm{msec}$ for all types of traffic. The packets arrive at an interval of $T_{a}^{k, i}=1 \mathrm{msec}$. The packet arrival rates of voice and $\mathrm{BE}$ traffic are constantly $\lambda_{k, i}^{\text {voice }}=64 \mathrm{Kbps}$ and $\lambda_{k, i}^{B E}=350 \mathrm{Kbps}$, respectively. The packet arrival rate of

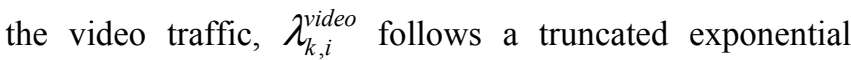
distribution in each state, with the maximum, minimum, and mean of $420 \mathrm{Kbps}, 120 \mathrm{Kbps}$ and $239 \mathrm{Kbps}$, respectively, where the duration of each state follows an exponential distribution with mean $160 \mathrm{msec}$. The average packet sizes for voice, video, and BE traffic can be calculated as 64 bits, 239 bits and 350 bits, respectively. The QoS priority levels for the voice, video, and $\mathrm{BE}$ traffic are respectively set to be $\beta_{k, i}^{\text {voice }}=1024, \beta_{k, i}^{\text {video }}=512$ and $\beta_{k, i}^{B E}=1$ as a result of testing, to provide the best performance. Based on different requirements of different traffic types on performance and complexity, the number of voice, video, and BE packets used for weight calculation in (9) are set to be $\Lambda_{k, i}^{\text {voice }}=100$, $\Lambda_{k, i}^{\text {video }}=75$ and $\Lambda_{k, i}^{B E}=50$, respectively. Here $k \in$ $\{1,2, \ldots, K\}, n \in\{1,2, \ldots, N\}$ and $i \in\left\{1,2, \ldots, I_{k}\right\}$.

The wireless channel is modeled as a frequency-selective channel consists of six independent Rayleigh fading paths with an exponentially delay profile and a root-mean-square (RMS) delay spread of $0.5 \mu \mathrm{sec}$. The signal-to-noise ratio (SNR) is defined as the average ratio of the received signal power to noise power for each user.

Simulation results show the performance comparison of the proposed fair MWSC based resource allocation and MWSC resource allocation without fairness. Figures demonstrate the impact of number of users on performance of both algorithms with $\mathrm{SNR}=20 \mathrm{~dB}$.

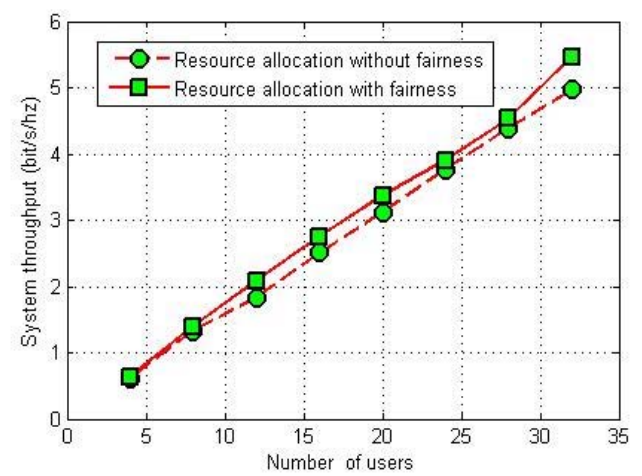

Fig. 2. Impact of the number of users on system throughput.

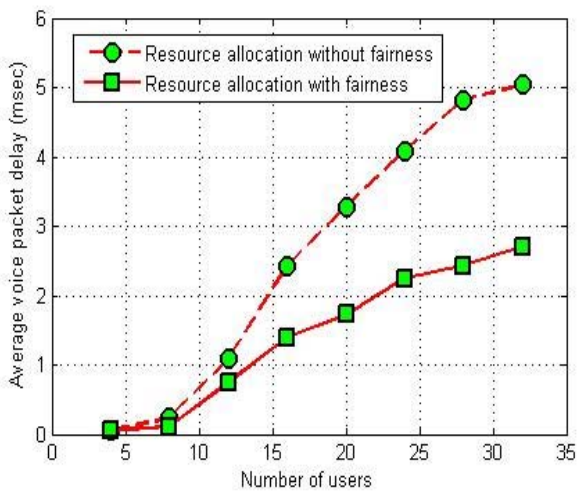

Fig. 3. Impact of the number of users average voice packet delay.

Fig. 1 shows the impact on system throughput. Fair MWSC resource allocation algorithm has better throughput than MWSC resource allocation algorithm without fairness. Throughput is increased by $9 \%$ for 16 users. When the number of users is 20 it is increased by $8 \%$ and for 32 users it is increased by $9 \%$.

The system throughput increases with the number of users due to enhanced multiuser diversity. When the number of users increases, there is a high degree of freedom for resource allocation, which increases the multiuser diversity. On the other hand, the increase in the number of users would cause the increase in weight difference of the HOL packets of various queues, and it decreases the system throughput.

Fig. 2 shows the impact of the number of users on the average voice traffic delay. Fair MWSC based resource allocation algorithm achieves a lower voice delay than MWSC based resource allocation algorithm without fairness. Average voice packet delay for the proposed algorithm is lower than the fair MWSC resource allocation algorithm. When the number of users is 16 then average voice delay is reduced by $41.6 \%$, when the number of users is 24 then it is reduced by $44 \%$, and for 32 users it is reduced by $46 \%$.

A similar trend can be observed in Fig. 3 for the average video traffic delay. Average video packet delay is also reduced for the proposed algorithm. Average video packet delay for the proposed algorithm is also lower than the fair MWSC resource allocation algorithm. When the number of users is 16 then average voice delay is reduced by $9.5 \%$, when the number of users is 24 then it is reduced by $11 \%$, 
for 32 users it is reduced by $10 \%$.

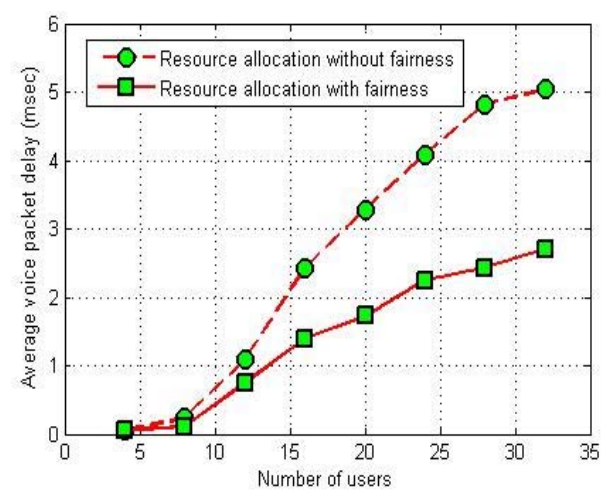

Fig. 4. Impact of the number of users average video packet delay.

\section{CONCLUSION}

We have proposed an adaptive fair MWSC based resource allocation and packet dependent scheduling algorithm using cross-layer design for the multiuser OFDM systems in which each user is having multiple heterogeneous traffic queues. This algorithm also encompasses the fairness; hence all the users will get the data rate in proportion of their weights. This algorithm maximizes the weighted sum capacity while maintaining proportional fairness. The proposed fair MWSC resource allocation algorithm achieves better performance than the previously proposed MWSC resource allocation algorithm without fairness [4]. It has higher throughput performance and lower average voice and video packet delays. It has lower complexity than optimal subcarrier allocation algorithm but the inclusion of fairness increases the overall complexity (for comparisons) of proposed algorithm in comparison to algorithm proposed in [4]. Complexity of comparisons of subcarrier allocation step increases to $\mathrm{O}\left(\mathrm{N}^{2}\right)$ from $\mathrm{O}(\mathrm{KN})$ while the complexity of power allocation remains same. A packet dependent scheduling is also employed at MAC layer which transmits the packet in the order of their weights. It achieves better performance than queue based scheduling. This user based cross-layer design also achieves lower complexity than the conventional queue based designs.

\section{ACKNOWLEDGMENT}

The authors are thankful to ABV-Indian Institute of Information Technology and Management Gwalior, India for extending the financial support for this paper.

\section{REFERENCES}

[1] J. G. Andrews, A. Ghosh and R. Muhamed, Fundamentals of WiMAX, Prentice Hall, 2007, ch. 4, pp. 113-117.

[2] G. C. Song and Y. Li, "Utility-based resource allocation and scheduling in OFDM-based wireless broadband networks," IEEE Communication Magazine, vol. 43, pp. 127-134, Dec. 2005.

[3] J. Cai, X. Shen and J. W. Mark, "Downlink resource management for packet transmission in OFDM wireless communication systems," IEEE Transactions on Wireless Communications $\}$, vol. 4, no. 4, pp. 1688-1703, Jul. 2005.

[4] N. Zhou, X. Zhu, Y. Huang and H. Lin, "Low complexity cross-layer design with packet dependent scheduling for heterogeneous traffic in multiuser OFDM systems," IEEE Transactions on Wireless Communications, vol. 9, no. 6, pp. 1912-1923, Jun. 2010.
[5] Z. Kong, Y. Kwok and J. Wang, "A low complexity QoS aware proportional fair multicarrier scheduling algorithm for OFDM systems," IEEE Transactions on Vehicular Technology, vol. 58, no. 5, pp. 2225-2235, Jun. 2009.

[6] Z. K. Shen and J. G. Andrews, "Adaptive resource allocation in multiuser OFDM systems with proportional rate constraints," IEEE Transactions on Wireless Communications, vol. 4, pp. 2726-2737, Nov. 2005.

[7] C. Y. Wong, R. S. Cheng, K. B. Letaief, and R. D. Murch, "Multiuser OFDM with adaptive subcarrier, bit and power allocation," IEEE JSAC, vol. 17, pp. 1747-1758, Oct. 1999.

[8] C. Anton-Haro, P. Svedman, M. Bengtsson, A. Alexiou, and A. Gameiro, "Cross-layer scheduling for multi-user MIMO systems," IEEE Commun. Mag., vol. 44, pp. 39-45, Sept. 2006.

[9] G. C. Song, "Cross-layer resource allocation and scheduling in wireless multicarrier networks," Ph.D. dissertation, Georgia Institute of Technology, 2005.

[10] M. Andrews, K. Kumaran, K. Ramanan, A. Stolyar, P. Whiting, and R. Vijayakumar, "Providing quality of service over a shared wireless link," IEEE Commun. Mag., vol. 2, pp. 150-154, Feb. 2001.

[11] S. Ryu, B. Ryu, H. Seo, and M. Shin, "Urgency and efficiency based packet scheduling algorithm for OFDMA wireless system," in Proc. IEEE ICC' 2005, Seoul, South Korea, 2005.

[12] J. Jang and K. B. Lee, "Transmit power adaptation for multiuser OFDM systems," IEEE J. Sel. Areas Commun., vol. 21, pp. 171-178, Feb. 2003.

[13] D. Veronesi, S. Tomasin, and N. Benvenuto, "Cross-layer optimization for multimedia traffic in CDMA cellular networks," IEEE Trans. Wireless Commun., vol. 7, pp. 1379-1388, Apr. 2008.

[14] K. B. Johnsson and D. C. Cox, "An adaptive cross layer scheduler for improved QoS support of multiclass data services on wireless systems," IEEE J. Sel. Areas Commun., vol. 23, pp. 334-343, Feb. 2005.

[15] Y. Yu and W. Zhou, "Resource allocation for OFDMA system based on genetic algorithm," in Proc. IEEE IWCLD' 2007, vol. 10, Tai'an, China, Sep. 2007, pp. 65-69.

[16] S. Boyd and L. Vandenberghe, Convex Optimization, Cambridge University Press, 2004.

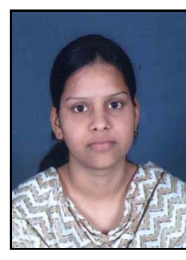

Kalpana Goyal received the B.E. degree in Electronics and Communication Engineering from Rajiv Gandhi Proudyogiki Vishwavidyalaya, Bhopal in 2008 and M.Tech. degree in Digital Communication from ABV-Indian Institute of Information Technology and Management, Gwalior, India in 2011.

She is now an Assistant Professor in Maulana Azad National Institute of Technology, Bhopal, India. Her research interests include MIMO OFDM systems, cross layer design, wireless communications etc.

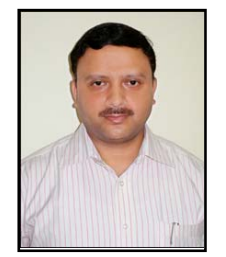

Dr. Aditya Trivedi is a Professor in the ICT Department of ABV-Indian Institute of Information Technology and Management, Gwalior, India. He received his bachelor degree (with distinction) in Electronics Engineering from the Jiwaji University and M.Tech. degree (Communication Systems) from Indian Institute of Technology (IIT), Kanpur. He obtained his doctorate $(\mathrm{PhD})$ from IIT Roorkee in the area of Wireless Communication Engineering.

His teaching and research interest include Digital communication, CDMA systems, Signal processing, and Networking. He is a fellow of the Institution of Electronics and Telecommunication Engineers (IETE) and a member of Institution of Electrical and Electronics Engineers (IEEE),USA. He has published more than 50 papers in various prestigious International/National journals and conferences. In 2007, he was given the IETE's K.S. Krishnan Memorial Award for best system oriented paper.

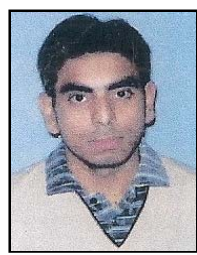

Karan Nathwani received the B.Tech degree in Electronics \& Communication from UPTU University in 2009 and M.Tech. degree in Digital Communication from ABV-IIITM Gwalior in 2011.

$\mathrm{He}$ is now a Ph. D. candidate in Electronics department in IIT Kanpur. His research interests include speech processing, wireless communication etc. 